\title{
3efferson.
}

JHN Journal

Volume 13 | Issue 1

Article 2

Winter 2018

\section{Phase 1 Trial of Vaccination with Autologous Tumor Cells and Antisense Directed Against the Insulin Growth Factor Type 1 Receptor (IGF-1R AS ODN) in Patients with Recurrent Glioblastoma}

David W. Andrews MD

Thomas Jefferson University, david.andrews@jefferson.edu

Kevin Judy, MD

Thomas Jefferson University, Kevin.Judy@jefferson.edu

Larry Harshyne, $\mathrm{PhD}$

Thomas Jefferson University, larry.harshynejr@jefferson.edu

D. Craig Hooper, $\mathrm{PhD}$

Thomas Jefferson University, douglasc.hooper@jefferson.edu

Follow this and additional works at: https://jdc.jefferson.edu/jhnj

\section{Let us know how access to this document benefits you}

\section{Recommended Citation}

Andrews MD, David W.; Judy, MD, Kevin; Harshyne, PhD, Larry; and Hooper, PhD, D. Craig (2018) "Phase 1 Trial of Vaccination with Autologous Tumor Cells and Antisense Directed Against the Insulin Growth Factor Type 1 Receptor (IGF-1R AS ODN) in Patients with Recurrent Glioblastoma," JHN Journal: Vol. 13 : Iss. 1 , Article 2.

DOI: https://doi.org/10.29046/JHNJ.013.1.002

Available at: https://jdc.jefferson.edu/jhnj/vol13/iss1/2

This Article is brought to you for free and open access by the Jefferson Digital Commons. The Jefferson Digital Commons is a service of Thomas Jefferson University's Center for Teaching and Learning (CTL). The Commons is a showcase for Jefferson books and journals, peer-reviewed scholarly publications, unique historical collections from the University archives, and teaching tools. The Jefferson Digital Commons allows researchers and interested readers anywhere in the world to learn about and keep up to date with Jefferson scholarship. This article has been accepted for inclusion in JHN Journal by an authorized administrator of the Jefferson Digital Commons. For more information, please contact:

JeffersonDigitalCommons@jefferson.edu. 


\section{Phase 1 Trial of Vaccination with Autologous Tumor Cells and Antisense Directed Against the Insulin Growth Factor Type 1 Receptor (IGF-1R AS ODN) in Patients with Recurrent Glioblastoma}

\author{
David W. Andrews, $M D^{1}$; Kevin D. Judy, MD; Larry A. Harshyne, PhD; \\ D. Craig Hooper, $\mathrm{PhD}^{2}$ \\ ${ }^{1}$ Department of Neurological Surgery, Thomas Jefferson University, Philadelphia, PA \\ 2Department of Cancer Biology, Thomas Jefferson University, Philadelphia, PA
}

\begin{abstract}
Background: Extending a previous Phase I study, we report the results of a second Phase I autologous tumor cell vaccination trial for patients with recurrent glioblastomas (IND 14379-101, NCT01550523).
\end{abstract}

Methods: Following surgery, subjects were treated by 24 hour implantation in the rectus sheath of ten biodiffusion chambers containing irradiated autologous tumor cells and IGF-1R AS ODN with the objective of stimulating tumor immunity. Patients were monitored for safety, clinical and radiographic as well as immune responses.

Results: There were no Grade 3 toxicities related to protocol treatment and overall median survival from initial diagnosis was 91.4 weeks. Two protocol survival cohorts with median survivals of 48.2 and 10 weeks were identified and predicted by our pre-treatment assessments of immune function, corroborated by post-vaccination pro-inflammatory cytokine profiles. Longer survival subjects had imaging findings including transient elevations in cerebral blood volume ( $\mathrm{rCBV}$ ) and sustained elevations of apparent diffusion coefficient (ADC) interpreted as transient hyperemia and cell loss.

Conclusions: The vaccine paradigm was well-tolerated with a favorable median survival. Our data support this as a novel treatment paradigm that promotes anti-tumor immunity.

\section{KEYWORDS}

Decompressive Hemicraniectomy, Intracerebral Hemorrhage, Malignant MCA Stroke, Traumatic Brain Injury, Aneurysmal Subarachnoid Hemorrhage, Intracranial Pressure, Herniation

\section{INTRODUCTION}

WHO Grade IV astrocytoma (glioblastoma) is a uniformly fatal primary intracranial malignancy with a median survival of 14 months. ${ }^{1}$ We conducted an earlier pilot Phase I vaccine trial in patients with high grade astrocytomas ${ }^{2}$ and designed a replacement Phase 1 trial with optimized reagents, expanded radiographic response criteria, and new exploratory objectives.

\section{METHODS}

\section{Study Site and Patients}

The study was conducted at Thomas Jefferson University and reached target accrual of 12 patients in 14 months. Criteria for enrollment included age $>18$, a Karnofsky performance score of 60 or better, and no co-morbidities that would preclude elective surgical re-resection. Twelve patients were enrolled for treatment after failure from standard therapy. ${ }^{1} \mathrm{~A}$ summary of enrolled patients, and all available pertinent data is included in Table 1.

\section{Study Design and Objectives}

As before, the combination product consisted of autologous tumor cells removed at surgery then treated overnight with the IGF-1R AS ODN $(4 \mathrm{mg} / \mathrm{ml})$ prior to being added to semi-permeable chambers and irradiated. ${ }^{2}$ Enhancements to the vaccine product included use of an 18-mer IGF-1R AS ODN with the sequence 5'-TCCTCCGGAGCCAGACTT-3', two frameshifts upstream from the previous sequence; and, based on data showing that the AS ODN has immunomodulatory properties, ${ }^{3,4}$ addition of $2 \mathrm{mg}$ of exogenous antisense to the chambers $(\mathrm{C}-\mathrm{v})$. The protocol was also amended to include an eleventh control chamber containing PBS ( $C-p)$. Study objectives included assessment of safety and radiographic responses as well as exploratory objectives looking at immune function and response.

\section{Radiological Assessments}

Serial imaging assessments were performed on Philips 1.5T and 3T MRIs and GE 1.5 T MRIs. Routine anatomic MRI features were evaluated as well as physiologic measurements including dynamic susceptibility weighted (DSC) MR perfusion and 15-direction diffusion tensor imaging (DTI) were also utilized.

\section{Immunological Assessments}

Plasma leukopheresis was performed one week before surgery for baseline assessment of immune function. Blood 
Table 1. Summary of Patients Enrolled.

\begin{tabular}{|c|c|c|c|c|c|c|c|c|}
\hline Subject & Age & KPS & $\begin{array}{l}\text { Interval between } \\
\text { surgeries (weeks) }\end{array}$ & $\begin{array}{l}\text { \# chambers } \\
\text { implanted }\end{array}$ & $\begin{array}{l}\text { Original lymphocyte } \\
\text { count (cells/mm2) }\end{array}$ & $\begin{array}{l}\text { Lymphocyte count } \\
\text { at enrollment } \\
\text { (cells/mm2) }\end{array}$ & $\begin{array}{l}\text { Previous } \\
\text { treatments }\end{array}$ & $\begin{array}{l}\text { IDH-1 mutation/ } \\
\text { MGMT methylation }\end{array}$ \\
\hline TJ01 & 39 & 70 & 177 & 10 & $N / A$ & 400 & S,RT + TMZ, Bev & -1 \\
\hline TJ02 & 57 & 80 & 90 & 9 & N/A & 1570 & $S, R T+T M Z$ & -/methylated \\
\hline TJ03 & 75 & 70 & 32 & 7 & 700 & 300 & $S, R T+T M Z$ & -1 \\
\hline TJ06/R1 & 66 & 80 & 54 & 8 & 2000 & 1300 & S,RT + TMZ & -1 \\
\hline TJ07 & 43 & 80 & 215 & 10 & 500 & 430 & $\begin{array}{c}\text { S, RT + TMZ, } \\
\text { Bev; RTOG } 0525\end{array}$ & +1 \\
\hline TJ08 & 55 & 80 & 52 & 8 & 1000 & 500 & $\mathrm{~S}, \mathrm{RT}+\mathrm{TMZ}$ & -1 \\
\hline TJ09 & 57 & 80 & 61 & 7 & 1400 & 300 & $\begin{array}{l}\text { S, RT + TMZ, } \\
\text { RTOG } 0929\end{array}$ & -/unmethylated \\
\hline TJ10 & 47 & 60 & 376 & 7 & N/A & 1800 & $\mathrm{~S}, \mathrm{RT}+\mathrm{TMZ}, \mathrm{Bev}$ & -/methylated \\
\hline TJ11 & 39 & 70 & 32 & $11^{*}$ & 2400 & 200 & S, RT + TMZ & -1 \\
\hline TJ12 & 60 & 80 & 74 & 7 & 1100 & 600 & $\begin{array}{l}\text { S, RT + TMZ, } \\
\text { Panobinostat }\end{array}$ & -1 \\
\hline TJ13 & 64 & 80 & 182 & 11 & $N / A$ & 2100 & $\mathrm{~S}, \mathrm{RT}+\mathrm{TMZ}$ & -1 \\
\hline TJ14/R & 77 & 90 & 30 & $7 / 11$ & 1800 & 1100 & S, RT + TMZ & -/unmethylated \\
\hline
\end{tabular}

was obtained post-operatively on days $7,14,28,42,56$, and every 3 months after vaccination. Sera and cell fractions were separated by centrifugation and cells were treated with red blood cell lysis buffer and white blood cells either quantified by flow cytometry or stored in DMSO at $-80^{\circ} \mathrm{C}$. Serum samples were also stored at $-80^{\circ} \mathrm{C}$. Flow cytometry was performed as previously described ${ }^{5}$ using an EasyCyte 8HT (Millipore) and fluorescently-conjugated mAb specific for human CD4, CD8,CD11b, CD14, CD16, CD20, CD45, CD56, CD80, CD83, and CD 86 (all from BD Biosciences), and CD163 (R\&D Systems). Post- collection analysis was performed with FlowJo software (Tree Star Inc, Ashland, OR). Serum cytokine factors were quantified using Luminex bead arrays (human cytokine/chemokine panels I, II, and III from Millipore and HCMBMAG/ MILLIPLEX Mag Cancer multiplex assay (emdmillipore.com). This included 6 serum markers for glioma related to stem cell function including DKK-1, NSE, Osteonectin, Periostin, YKL-40, and TWEAK. T-cell stimulation was performed with phorbol 12-myristate,13 acetate (PMA) and ionomycin as previously described.6 PBMC from glioblastoma patients and normal donors were cultured for 24 hours at $37^{\circ} \mathrm{C}$ and 5\% CO2 in AIMV media containing $10 \%$ fetal bovine serum (Gibco). Nonadherent lymphocytes were transferred to new plates in order to eliminate non-specific effects of contaminating monocytes. Lymphocyte cultures were stimulated with phorbol 12-myristate 13-acetate (PMA, $100 \mathrm{ng} / \mathrm{ml}$ ) and ionomycin (0.1 mM, both from Sigma-Aldrich) for 18 hours. BD Golgiplug ${ }^{\mathrm{TM}}$ (1:1000, Brefeldin A, BD Bioscience) was added during the last 6 hours of culture to permit cytokine accumulation.

Tumor tissue sections were assessed by immunohistochemistry or immunofluorescence, adapting the method of Emoto7, for GFAP, IGF-1R, CD163, CD14, CD3, CD4, and CD8. Immunopositive cells were counted quantitatively with Aperio or qualitatively by an experienced neuropathologist (LEK) using an ordinal scale from 0 (no staining) to 6 (strong diffuse staining) with staining intensity rated as low, moderate and strong and staining patterns described as focal or diffuse.

Cytokine/chemokine levels in tumor cell supernatant were analyzed by Luminex kits as designated above. Membranes from paired vaccine and control chambers were embedded in paraffin for standard immunohistopathologic examination. Post-mortem autopsy was limited to examination of the brain and findings were compared to archival paraffin blocks of previously treated or untreated glioblastomas diagnosed at autopsy.

\section{Statistical Analysis}

The level of statistical significance between quantitative measures in different samples was determined by a two-tailed unpaired t-test or matched pairs t-test with $p<.05$. Survival analysis was performed by Kaplan-Meier analysis and significance established by log rank comparisons. All statistical analysis including mixture discriminant analysis was performed with JMP v. 11 software (SAS, North Carolina). 

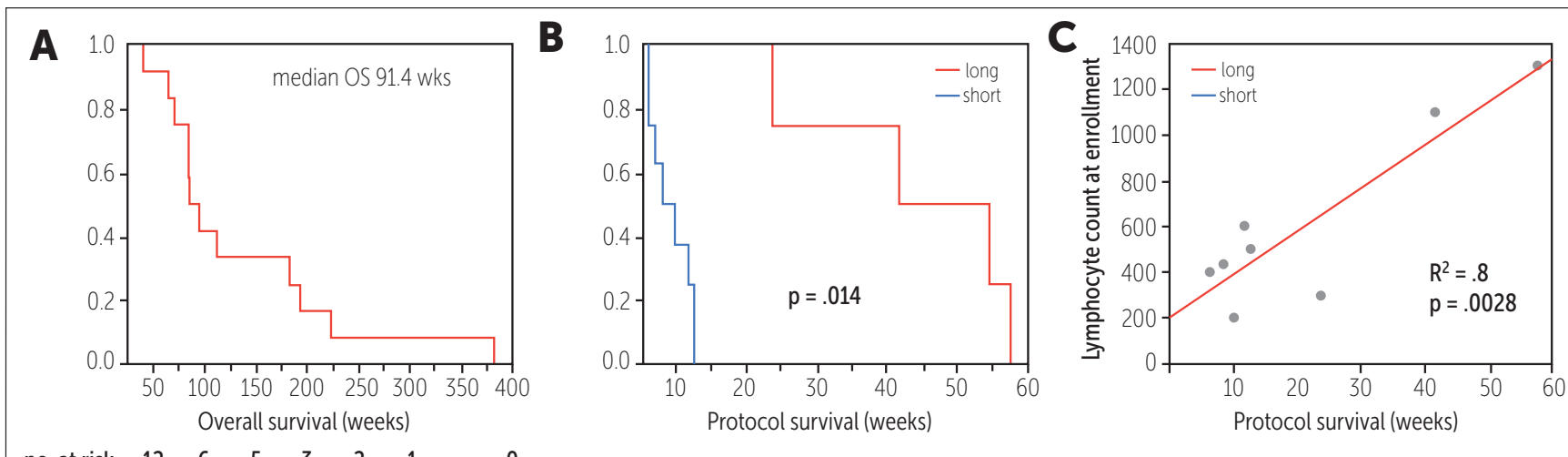

no. at risk $12 \quad 6 \quad 5 \quad 3 \quad 2 \quad 1 \quad 6$

\section{Figure 1. Survival Metrics.}

A. Overall survival of patients in trial; B. protocol survival with two survival cohorts; Nine patients died of disease progression while one died of intracerebral hemorrhage and two of sepsis. Overall protocol survival was 48.2 weeks and 9.2 weeks, respectively for longer $(\mathrm{N}=4)$ and short $(\mathrm{N}=8)$ survival cohorts (log-rank $=.014)$. C. Excluding one outlier and three non-disease-related deaths linear regression revealed high correlation between protocol survival and lymphocyte count at enrollment ( $R 2=.8, \mathrm{p}=.0028)$.

\section{RESULTS}

\section{Safety Assessment and Clinical Course}

Only one SAE was related to the protocol (femoral vein thrombosis after leukopheresis). Nine patients succumbed to tumor progression while three patients died from other causes. Five autopsies were performed.

Median overall survival from initial diagnosis was 91.4 weeks which compared favorably to other recurrent glioma immunotherapy trials. ${ }^{8-13}$ Two significantly different protocol survival cohorts of 48.2 and 10 weeks were identified as longer and short survival cohorts, respectively (Figure 1A\&B). Excluding one outlier (TJ03), we documented a significant correlation between protocol survival and degree of lymphopenia at enrollment (Figure 1C). Comparison of CBC values at initial diagnosis and at protocol enrollment indicated that the mean lymphocyte count had dropped significantly (65\%) after standard therapy $(\mathrm{N}=8, \mathrm{p}=.012$, paired t-test).

\section{Radiographic Responses}

Routine MRI features were assessed as previously described. ${ }^{2}$ In the longer survivors we noted diminished size of enhancement and FLAIR envelope at the primary tumor site and slower progression. Examples of anatomic responses in both cohorts is included noted in Figure 2A\&B. Physiologic MRI measurements augmented these anatomic observations for both cohorts is featured in Figure 2C\&D. Sequential DSC MR perfusion was performed in 7 patients, including 3 longer-term survivors (TJ03, TJ06, and TJ09) who had a paradoxical increase in rCBV while improving clinically; however, this effect was transient and there was a more sustained decrease in rCBV. Sequential 15 directions DTI data included two long-term survivors (TJ03 and TJ06) who showed ADC values increasing in the affected hemisphere, reflecting loss of tumor cellularity associated with disease regression.

\section{Immunologic Response Assessments after Vaccination by Survival Cohort}

Levels of 24 of the 78 cytokines/chemokines assessed were significantly higher in serum from the longer survival cohort compared to the short cohort. A spike in serum CCL2 occurred after surgery but was absent at re-operation in two patients. CCL2 levels remained significantly higher throughout the post-operative period in the short survival cohort. These postoperative spikes were highly correlated with TNF-a spikes. (data not shown).
Actual CD4 and CD8 T cell counts as well as DC counts were significantly higher in the longer cohort compared to the short cohort. There was a significant correlation between CD4 and DC cells and between CD4 and CXCL12 only in the longer cohort. Coordinated changes between circulating levels of T cells, monocytes, and pro-inflammatory chemokines/cytokines after vaccination were seen in three of four longer cohort subjects.

As a distinguishing feature differentiating the cohorts, peripheral blood cells from the longer survival subjects manifested significantly higher Th-1 cytokine production including IFN- $\gamma$ after stimulation with PMA and ionomycin from PBMC obtained on day 14

Levels of circulating lymphocytes were significantly decreased $(p<0.0001)$ in all GBM patients when compared to normal subject samples (Figure 1A, left panel) and the medians of both patient populations fell outside of the normal range (Figure $1 \mathrm{~A}$, gray lines). Patients in this same cohort exhibited significantly higher levels of monocytes (Figure 1A, right panel, $p<0.05$ ), but the medians of these cell populations fell within normal range.

The mere presence of a given cell subset is not enough to ensure proper immune function. The cell must also be able to respond to stimuli and produce 


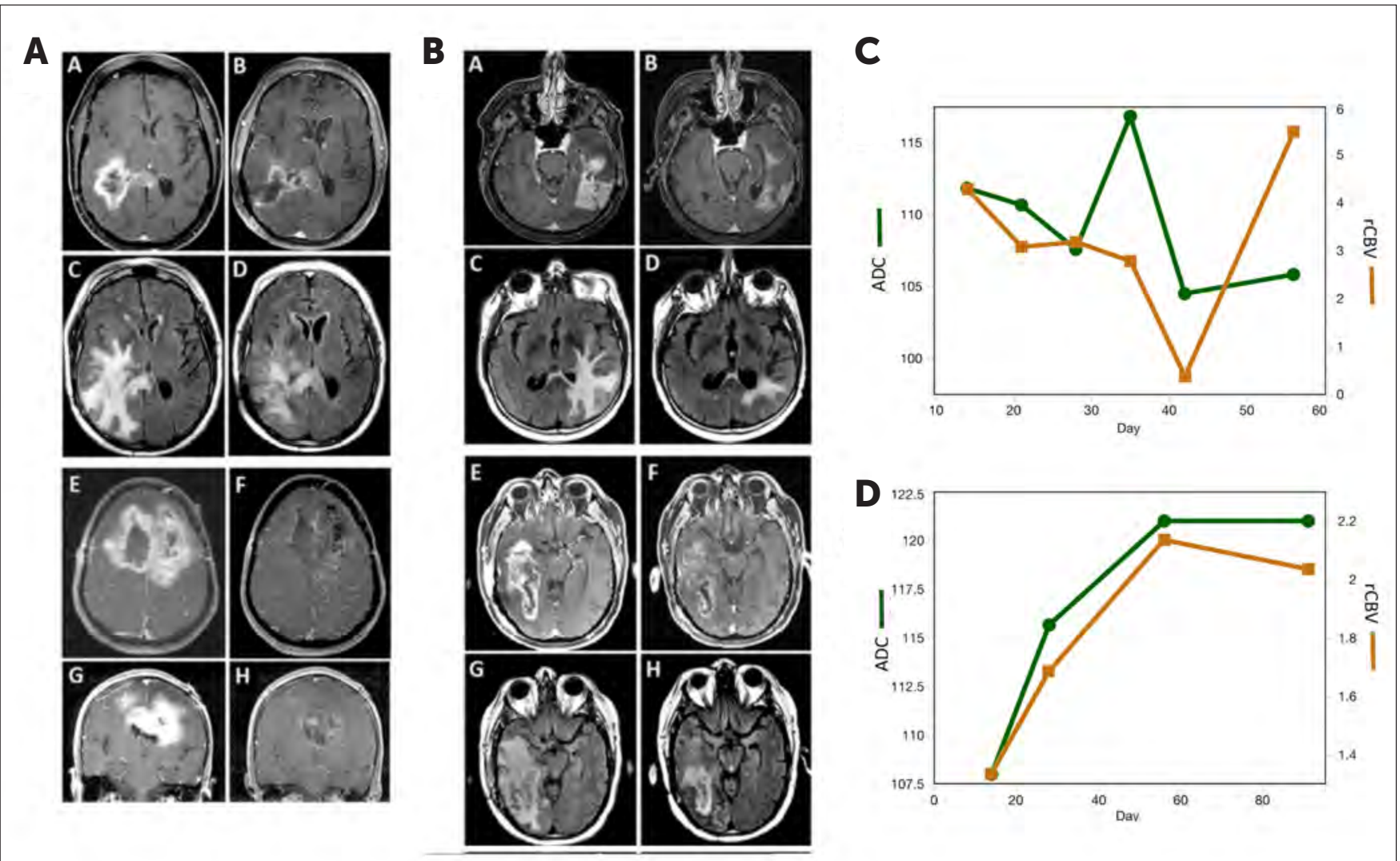

Figure 2. Radiographic responses with associated physiologic measurements and cytokine profiles.

A. Examples of short survival cohort. TJ11: A-D; TJ10: E-H. A,E: pre-operative T1-gadolinium-enhanced axial images; G: T1-gadoliniumenhanced coronal image; C: pre-operative axial FLAIR image. B,D,F,H: respective 3 month post-operative images. B. Examples of longer survival cohort. TJ06: A-D: TJ09: E-H. A,E: pre-operative T1-gadolinium-enhanced axial images; C,F: pre-operative axial FLAIR images. B,D,F,H: respective 3 month post-operative images. C. Relationship between relative cerebral blood volume in tumor $\mathrm{v}$. apparent diffusion coefficient in short survival cohort. D. Relationship between relative cerebral blood volume in tumor v. apparent diffusion coefficient in longer survival cohort; there is a high correlation between the $\operatorname{ADC}$ and $\mathrm{rCBV}(\mathrm{R} 2=.96, \mathrm{p}=.0005)$.

appropriate immune modulators. T-helper type 1 immunity is considered to be the appropriate anti-tumor immune response. Cultures of nonadherent lymphocytes were stimulated nonspecifically with phorbol 12-myristate 13-acetate (PMA) and ionomycin overnight in order to stimulate the production of IFN- $\gamma$, the prototypical Th1 cytokine which was detected by intracellular cytokine flow cytometry. Viable, CD3+ $T$ cell-specific gates were established (Figure 1B) and the median fluorescence intensity (MFI) of IFN- $\gamma+\mathrm{T}$ cells was normalized to $T$ cells that did not produce cytokine (IFN- $\gamma+$ T cells). PMA/ionomycin stimulation of normal $T$ cells resulted in a 26 -fold increase in the IFN- $\gamma$ MFI (Figure $1 B$, right panel). $T$ cells enriched from patients with primary and recurrent GBM produced significantly less IFN- $\gamma$ when compared to normal controls $(p<0.01)$, but there was no difference between GBM patient cohorts (Figure 1B, right panel).

Moreover, while a difference in the quantity of IFN- $\gamma$ produced by the $T$ cells was observed, there was no difference in the frequency of IFN $-\gamma+\mathrm{T}$ cells between these two cohorts (data not shown). In order to assess the relationship between lymphocyte numbers and immune functional capacity, we performed linear regression analyses. Lower levels of circulating lymphocytes are associated with a statistically significant $(R 2=0.508$, $\mathrm{p}=0.0093$ ) decrease in IFN $-\gamma$ production following PMA/ionomycin stimulation
(Figure 1C, left panel). Furthermore, recursive partitioning analyses identified two different populations ( $R 2=0.547)$ of recurrent GBM patients enrolled in our clinical trial (Figure $1 C$, right panel).

Reanalyzing these data focusing on single parameters based on the functional immune capacity confirmed the highly significant differences identified by recursive partitioning (Supplementary Figure 1). Patients with higher immune function possessed 3-fold higher levels of lymphocytes $(p<0.0001)$ and half as many monocytes $(p<0.005)$ when compared to the lower functioning group (Supplementary Figure 1). In addition to higher levels of lymphocytes, the T cells from patients with higher immune function produced twice as much IFN- $\gamma$ following stimulation 
A

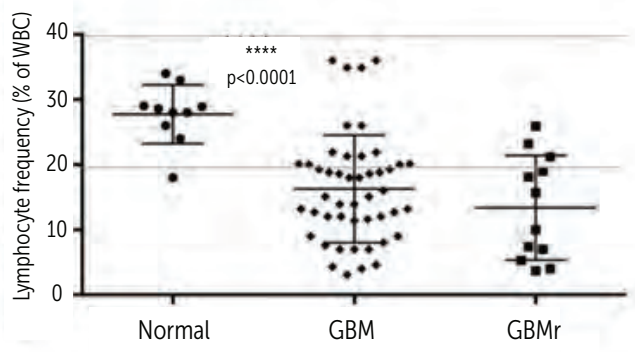

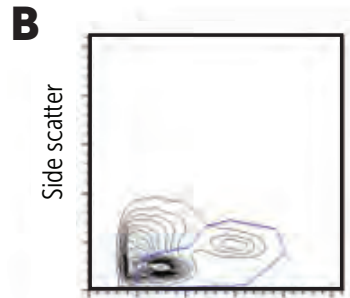

Forward scatter

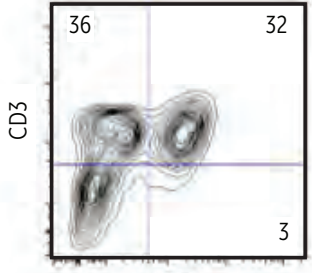

$\mathrm{IFN} \gamma$
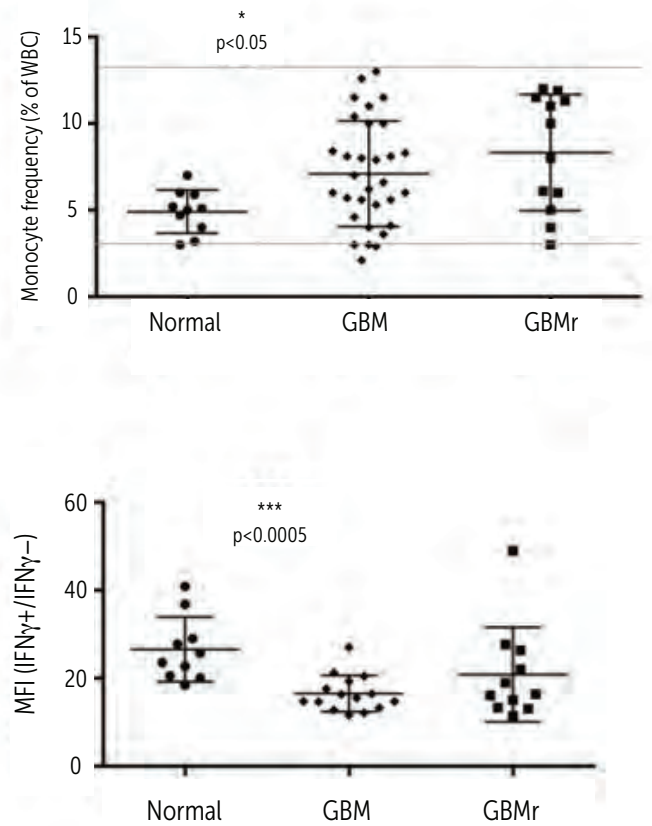

C

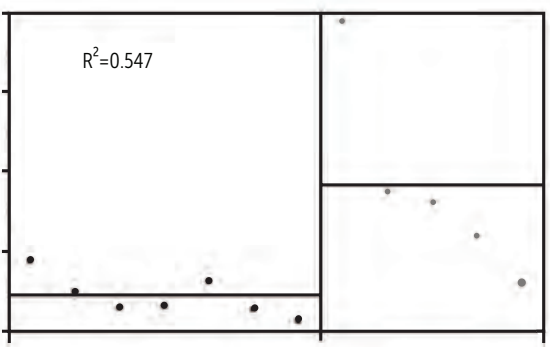

Lymphocytes $<15.7$
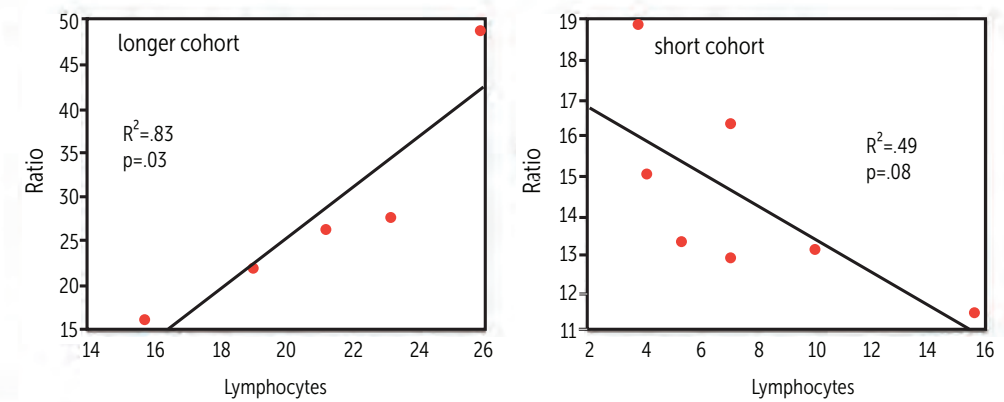

Figure 3. Functional immune capacity varies in glioblastoma patients.

A. Lymphocyte and monocyte frequencies are expressed as a percentage of white blood cells as determined by clinical blood counts performed by Thomas Jefferson University Hospital. Frequencies in trial patients are compared to primary glioblastoma patients and normal blood donors. Scatter dot plots indicate the mean frequency and standard deviation. Statistical significance of differences between tumor patients and normal blood donors were assessed by ANOVA followed by Dunnett's post-test (**** $p<0.0001$ and ${ }^{*} p<0.05$ ). Gray lines represent the upper and lower range of normal values. GBM signifies newly diagnosed patients; GBMr signifies recurrent glioblastoma patients. B. Non-adherent peripheral blood mononuclear cells were stimulated with PMA/ionomycin and cytokine production was assessed by intracellular flow cytometry. Live cell gates were established in forward- and side-scatter plots (left panel). Quadrant gates were used to assess IFN- $\gamma$ production in CD3+ T lymphocytes (middle panel). Numbers indicate the frequency of cells in a given quadrant. Median fluorescence intensity of IFN- $\gamma+\mathrm{CD} 3+$ cells was normalized to IFN- $\gamma-\mathrm{CD} 3+$ cells and is presented as a ratio in scatter dot plots (right panel). Statistical significance of the difference between tumor patients and normal blood donors was assessed by ANOVA followed by Dunnett's post-test $(* * *<0.0005)$. C. Left panel: Recursive partitioning analysis separated the trial patients into groups with higher and lower immune functional capacity (R2 $=0.547$ ). Right panel pair: Linear regression analysis of IFN $\gamma$ production versus peripheral blood lymphocyte frequency revealed a significant association of these variables only in the longer survival cohort with a positive correlation ( $R 2=0.83)$. 
with $\mathrm{PMA} /$ ionomycin (Supplementary Figure 1, $p<0.01$ ). Subsequent analyses will focus on patients with higher/lower functional immune capacity. Analysis of hospital-acquired blood cell counts fail to take in to consideration immune cell subsets. We performed flow cytometry phenotyping in order to further characterize the white blood cells. Frequencies of CD20+ B cells were significantly decreased in those patients with lower cohort $(p<0.005)$ and fell outside of the normal average (Figure 2). CD4+ T cells in both cohorts were significantly lower than the normal average, while CD14+ monocytes were significantly increased compared to normal averages (Figure 2) in both partitioned cohorts. CD8 + T cells in both cohorts were similar to normal averages (Figure 2). There was no difference between the lower and higher cohorts with respect to CD4+ or CD8+ T cell, or CD14+ monocyte frequencies (Figure 2).

\section{DISCUSSION}

Like its predecessor, the revised autologous cell/chamber-based GBM vaccination trial did not raise any significant safety concerns. We recognized, however, that an immunotherapy trial for recurrent GBM faces incumbent challenges. Patients emerging from standard therapy for GBM often have severe leukopenia, ${ }^{14}$ which was also documented in the current trial. It was therefore not surprising that we could not document $T$ cell infiltrates in the TME after treatment. Surgery itself enhances host immune mechanisms favoring tumor growth through the attraction of $\mathrm{M} 2$ macrophages to the tumor environment postoperatively ${ }^{15,16}$ suggested by the CCL2 serum spike provoked by craniotomy. Other than this post-operative serum spike, the highest levels of CCL2 expression were found in the tumor supernatants during vaccine preparation supporting well-documented observations that CCL2 is produced from cells in the TME. ${ }^{17,18}$ The lack of a CCL2 peak after re-vaccination in two patients suggested loss of these cells after the first vaccination.

We identified two significantly different survival cohorts with different responses to this vaccine paradigm allowing us to explore the nature of potentially therapeutic immune mechanisms. Serum from the longer cohort subjects contained higher levels of tumor-specific antibody isotypes and cytokines/chemokines commonly associated with Th1 immunity including IgG1, IgG3, IL12, CXCL10, CXCL12, CCL7, CCL19, and CCL21 not seen in the short cohort.

The short survival cohort had longer overall survival perhaps in part due to MGMT methylation and better responses to temozolamide in three patients. However the treatment-induced lymphopenia and the lower CD4:CD8 ratio could perhaps also be ascribed to temozolamide. Also, elevated serum CCL2 levels found in the short cohort have been associated with the mesenchymal gene expression profile ${ }^{19}$ and a poor prognosis ${ }^{20}$ in glioma patients.

Cytokine production is a hallmark feature of immune function in lymphocytes. We used phorbol ester-mediated, non-specific stimulation of patient lymphocytes in order to stimulate maximal cytokine output as a surrogate indicator of immune function. IFN- $\gamma$ is the classical Th1 cytokine and the logical choice for assessing immune fitness of $T$ lymphocytes in our GBM patients. The amount of IFN- $\gamma$ production by $T$ cells following overnight stimulation was approximately half the amount produced by normal donor cells treated similarly. IFN- $\gamma$ production and lymphocyte counts were strongly associated and predictively identified patients with different levels of immune fitness that fell into either the longer or short survival cohort. Lymphocytes from patients with higher functional immune capacity were more frequent and produced higher levels of IFN- $\gamma$ that approached levels to those produced by normal lymphocytes stimulated under similar conditions. In contrast, T cells from trial patients in the lower immune function cohort were less in number $(50 \%)$ and unable to respond to PMA stimulation.

The implanted chambers have inherent adjuvanticity. We have confirmed that the antisense sequence, its CpG motif, and the direct mixture with glioma cells in situ effectively initiate anti-tumor immunity ${ }^{3}$ also noted by others. ${ }^{21,22}$ The CpG motif specifically interacts with TLR94 causing plasmacytoid DC activation, measured by CD80 and CD86 expression. ${ }^{5}$ The elevated CD4:CD8 ratio after vaccination in the longer survival cohort could reflect local TLR9 DC activation and CD4 T cell stimulation.
Evolution of resistance to treatments in gliomas has now been associated with activation of the IGF-1R signaling axis and IGF-1R inhibition through a small molecule inhibitor overcomes this resistance with improved outcomes. ${ }^{23}$ We are currently exploring the impact of the IGF-1 bioregulatory system in recurrent gliomas and the impact of the IGF-1R AS ODN in this vaccination paradigm.

Differences in the radiographic observations between the longer and short survival patient cohorts provide further support for the concept that the vaccination paradigm may have an impact on the glioma TME. Higher rCBV values are typically associated with tumor progression, ${ }^{24}$ and MR perfusion had only transient increases in the longer cohort, a finding not previously described. ADC measurements differentiated tumor progression (lower values) from what we interpreted as cell loss (higher values). ${ }^{25}$

In summary, we have established the safety profile of an improved combination glioma vaccine product and have documented alterations in immune parameters associated with clinical and radiographic improvements. Despite immune compromise, we documented favorable immune responses associated with tumor regression and longer survival after treatment. To be most effective, however, a replacement trial should include screens for immune compromise and means by which immune function could be restored prior to vaccination.

\section{Study Oversight}

The physician sponsor (DWA) was primarily responsible for the design and funding of the study. All authors participated in the conduct of the study, analysis of the data and the reporting of the results. After IRB approval, this study was overseen by an independent data safety and monitoring board appointed by the Sidney Kimmel Cancer Center at Thomas Jefferson University.

DWA and DCH have financial interests in the Imvax Corporation related to this work. 


\section{REFERENCES}

1. Stupp R, Mason WP, van den Bent MJ, et al: Radiotherapy plus concomitant and adjuvant temozolomide for glioblastoma. N Engl J Med 352:987-96, 2005

2. Andrews DW, Resnicoff $M$, Flanders $A E$, et al: Results of a pilot study involving the use of an antisense oligodeoxynucleotide directed against the insulin-like growth factor type I receptor in malignant astrocytomas. J Clin Oncol 19:2189-200, 2001

3. Morin-Brureau M, Hooper KM, Prosniak $M$, et al: Enhancement of glioma-specific immunity in mice by "NOBEL", an insulin-like growth factor 1 receptor antisense oligodeoxynucleotide. Cancer Immunol Immunother 64:447-57, 2015

4. Agrawal S, Kandimalla ER: Role of Toll-like receptors in antisense and siRNA [corrected]. Nat Biotechnol 22:1533-7, 2004

5. Harshyne LA, Hooper KM, Andrews EG, et al: Glioblastoma exosomes and IGF-1R/AS-ODN are immunogenic stimuli in a translational research immunotherapy paradigm. Cancer Immunol Immunother 64:299-309, 2015

6. Verbrugge I, Hagekyriakou J, Sharp LL, et al: Radiotherapy increases the permissiveness of established mammary tumors to rejection by immunomodulatory antibodies. Cancer Res 72:3163-74, 2012

7. Emoto K, Yamashita S, Okada Y: Mechanisms of heat-induced antigen retrieval: does $\mathrm{pH}$ or ionic strength of the solution play a role for refolding antigens? J Histochem Cytochem 53:1311-21, 2005

8. Bloch $O$, Crane CA, Fuks Y, et al: Heat-shock protein peptide complex-96 vaccination for recurrent glioblastoma: a phase II, single-arm trial. Neuro Oncol 16:274-9, 2014

9. Kikuchi $T$, Akasaki $Y$, Abe $T$, et al: Vaccination of glioma patients with fusions of dendritic and glioma cells and recombinant human interleukin 12. J Immunother 27:452-9, 2004
10. Okada H, Lieberman FS, Edington HD, et al: Autologous glioma cell vaccine admixed with interleukin-4 gene transfected fibroblasts in the treatment of recurrent glioblastoma: preliminary observations in a patient with a favorable response to therapy. J Neurooncol 64:13-20, 2003

11. Prins RM, Soto $H$, Konkankit V, et al: Gene expression profile correlates with T-cell infiltration and relative survival in glioblastoma patients vaccinated with dendritic cell immu notherapy. Clin Cancer Res 17:1603-15, 2011

12. Rutkowski S, De Vleeschouwer S, Kaempgen E, et al: Surgery and adjuvant dendritic cellbased tumour vaccination for patients with relapsed malignant glioma, a feasibility study. Br J Cancer 91:1656-62, 2004

13. Schuessler A, Walker DG, Khanna R: Cellular immunotherapy directed against human cytomegalovirus as a novel approach for glioblastoma treatment. Oncoimmunology 3:e29381, 2014

14. Grossman SA, Ye $X$, Lesser $G$, et al: Immunosuppression in patients with high-grade gliomas treated with radiation and temozolomide. Clin Cancer Res 17:5473-80, 2011

15. Hamard L, Ratel D, Selek L, et al: The brain tissue response to surgical injury and its possible contribution to glioma recurrence. J Neurooncol 128:1-8, 2016

16. Predina J, Eruslanov E, Judy B, et al: Changes in the local tumor microenvironment in recurrent cancers may explain the failure of vaccines after surgery. Proc Natl Acad Sci USA 110:E415-24, 2013

17. Desbaillets I, Tada M, de Tribolet N, et al: Human astrocytomas and glioblastomas express monocyte chemoattractant protein-1 (MCP-1) in vivo and in vitro. Int J Cancer 58:240-7, 1994

18. Leung SY, Wong MP, Chung LP, et al: Monocyte chemoattractant protein-1 expression and macrophage infiltration in gliomas. Acta Neuropathol 93:518-27, 1997
19. Engler JR, Robinson AE, Smirnov I, et al: Increased microglia/macrophage gene expression in a subset of adult and pediatric astrocytomas. PLoS One 7:e43339, 2012

20. Arimappamagan A, Somasundaram K, Thennarasu K, et al: A fourteen gene GBM prognostic signature identifies association of immune response pathway and mesenchymal subtype with high risk group. PLoS One 8:e62042, 2013

21. Wu A, Oh S, Gharagozlou S, et al: In vivo vaccination with tumor cell lysate plus $\mathrm{CpG}$ oligodeoxynucleotides eradicates murine glioblastoma. J Immunother 30:789-97, 2007

22. Morishita M, Takahashi Y, Matsumoto A, et al: Exosome-based tumor antigens-adjuvant co-delivery utilizing genetically engineered tumor cell-derived exosomes with immunostimulatory CpG DNA. Biomaterials 111:55-65, 2016

23. Quail DF, Bowman RL, Akkari L, et al: The tumor microenvironment underlies acquired resistance to CSF-1R inhibition in gliomas. Science 352:aad3018, 2016

24. Gasparetto EL, Pawlak MA, Patel SH, et al: Posttreatment recurrence of malignant brain neoplasm: accuracy of relative cerebral blood volume fraction in discriminating low from high malignant histologic volume fraction. Radiology 250:887-96, 2009

25. Hein PA, Eskey CJ, Dunn JF, et al: Diffusionweighted imaging in the follow-up of treated high-grade gliomas: tumor recurrence versus radiation injury. AJNR Am J Neuroradiol 25:201-9, 2004 